\title{
Synergistic Effect of Deoxyanthocyanins from Symbiotic Fern Azolla spp. on hrmA Gene Induction in the Cyanobacterium Nostoc punctiforme
}

\author{
Michael F. Cohen, ${ }^{1}$ Yasuko Sakihama, ${ }^{1}$ Yojiro C. Takagi, ${ }^{1}$ Toshio Ichiba, ${ }^{2}$ and Hideo Yamasaki ${ }^{1}$ \\ ${ }^{1}$ Laboratory of Cell and Functional Biology, Faculty of Science, University of the Ryukyus, Nishihara, Okinawa 903-0213, \\ Japan; ${ }^{2}$ Research \& Development Division, Okinawa Industrial Technology Center (OITC), 12-2 Suzaki, Gushikawa, \\ Okinawa 904-2234 Japan
}

Submitted 11 February 2002. Accepted 19 April 2002.

\begin{abstract}
The hrmA gene of the $\mathrm{N}_{2}$-fixing cyanobacterium Nostoc punctiforme functions in repressing the formation of transitory motile filaments, termed hormogonia, by plant-associated vegetative filaments. Here, we report that anthocyanins can contribute to induction of $h r m A$ expression. Aqueous extract from fronds of the fern Azolla pinnata, a host of symbiotic Nostoc spp., was found to be a potent inducer of hrmA-luxAB in N. punctiforme strain UCD 328. The hrmA-luxAB inducing activities of $A$. pinnata, as well as Azolla filiculoides, were positively correlated with levels of frond deoxyanthocyanins. Analyses of the deoxyanthocyanins in frond extracts revealed, in order of predominance, an acetylated glycoside derivative of luteolinidin $(\mathrm{m} / \mathrm{z} 475)$ and of apigeninidin $(\mathrm{m} / \mathrm{z}, 459)$ and minor amounts of a second luteolinidin derivative. At up to $150 \mu \mathrm{M}$, a purified preparation of deoxyanthocyanins only weakly induced hrmA-luxAB on its own, but mixtures with hrmAlux $A B$ inducers (A. filiculoides extract or the flavonoid naringin) synergistically doubled to tripled their inducing activities. These results suggest that appropriately localized deoxyanthocyanins could function in plant-mediated mechanisms for repressing Nostoc spp. hormogonium formation.
\end{abstract}

Additional keywords: symbiosis, Anthoceros, cycad, Gunnera, heterocyst, rhizobia.

Filamentous $\mathrm{N}_{2}$-fixing cyanobacteria of the genus Nostoc form associations with a diverse array of plant species (Dodds et al. 1995; Meeks 1998; Rai et al. 2000). Nostoc spp. infect plant host tissue as temporarily differentiated, typically motile, filaments termed hormogonia. Although hormogonia are formed at a low frequency as part of the Nostoc spp. life cycle, host control over the timing of their development is essential for establishing efficient symbioses (Meeks 1998). Under low $\mathrm{N}$ conditions certain plants exude one or more unidentified HIF, dramatically increasing the frequency at which nearby Nostoc spp. filaments convert to hormogonia (Campbell and Meeks 1989; Gantar et al. 1993). Some of these hormogonia may then locate and infect plant symbiotic regions, presumably by chemotaxis (Meeks 1998). The hormogonium state is necessarily transient since no growth or $\mathrm{N}_{2}$-fixation occurs (Campbell and Meeks 1989). Accordingly, successful colonization of plants

Corresponding author: H. Yamasaki; Telephone: +81-98-895-8550; Fax: +81-98-895-8576; E-mail: yamasaki@comb.u-ryukyu.ac.jp. appears to require that newly attached Nostoc spp. filaments repress hormogonium formation even in an HIF-rich environment (Cohen and Meeks 1997; Gantar et al. 1993).

Recently, methods for molecular investigation of Nostoc punctiforme symbiotic interactions have been developed using Nostoc punctiforme ATCC 29133 as a model organism (Cohen et al. 1994, 1998). N. punctiforme was originally isolated from symbiotic association with the cycad Macrozamia sp. and can reconstitute symbiotic associations with the angiosperm Gunnera (Johansson and Bergman 1994) and the bryophyte Anthoceros punctatus (Campbell and Meeks 1989; Enderlin and Meeks 1983). Inactivation of the $N$. punctiforme hrmA gene by transposon or insertional mutagenesis results in a higher frequency of hormogonium formation in response to HIF of $A$. punctatus (Cohen et al. 1994; Cohen and Meeks 1997). The hrmA gene is part of a hrmRIUA operon that has high sequence similarity to sugar uronate metabolism operons of other bacteria (Meeks et al. 1999). N. punctiforme hrmA mutants are unable to survive in long-term coculture with $A$. punctatus due to their continued formation of hormogonia (Cohen and Meeks 1997). In contrast, wild-type Nostoc spp. filaments, after an initial burst of HIF-induced hormogonium formation, show a period of immunity to HIF that allows for vegetative growth and $\mathrm{N}_{2}$-fixation (Campbell 1988; Gantar et al. 1993). Extract of ground A. punctatus tissue prevents HIF-induced hormogonium formation (Cohen and Meeks 1997) and induces accumulation of $\mathrm{hrm}$ locus transcripts in wild-type $N$. punctiforme (E. L. Campbell and J. C. Meeks, personal communication) and has been shown to induce luciferase activity from a hrmAlux $A B$ transcriptional fusion in $N$. punctiforme::Tn5-1063 mutant strain UCD 328 (Cohen and Meeks 1997).

The symbiosis between the aquatic fern Azolla and N. punctiforme is of particular interest because it is the only plant-prokaryote symbiosis known to persist throughout the reproductive cycle of the host plant. Vegetative maintenance of the association depends on the retention of filaments, morphologically similar to hormogonia, at the apical meristem of fronds (Peters and Meeks 1989). Epidermal trichomes (or "hair cells") assist in partitioning these filaments into developing symbiotic cavities (Calvert et al. 1985). As in other Nostoc spp.-plant symbioses, hormogonium formation in mature cavities appears to be blocked and the Nostoc spp. vegetative filaments instead differentiate a higher than free-living frequency of $\mathrm{N}_{2}$-fixing cells, termed heterocysts, that can provide the association with its total $\mathrm{N}$ requirement (Meeks 1998).

Reasoning that repression of hormogonium formation in Azolla leaf cavities may act via induction of the hrm operon, 
we have examined in this study the effect of Azolla extract on luciferase activity from cells of strain UCD 328. We focused on the involvement of Azolla flavonoids in hrmA-luxAB induction, since flavonoids are common indicators to other organisms of plant proximity (Cohen et al. 2001) and because a flavonoid, naringin, is as yet the only compound found to induce hrmA at substantial levels (Cohen and Yamasaki 2000). The only known flavonoids of Azolla are the colored 3-deoxyanthocyanins (Harborne and Baxter 1999; Ishikura 1982; Peters and Meeks 1989). During winter season and under conditions of phosphate deficiency, Azolla fronds take on a reddish color due to increased accumulations of deoxyanthocyanins (Pieterse et al. 1977; Wagner 1997).

The widespread presence of various anthocyanins in plant tissues serves several adaptive purposes. They are best recognized for their role in flowers as visual cues for animals (Bohm 1998). In vegetative tissues, anthocyanins may contribute to protection from photoinhibition (Gould et al. 1995), tolerance to cold temperatures (Camm et al. 1993), scavenging of active oxygen species (Yamasaki 1997; Yamasaki et al. 1996), chelation of metallic pollutants, and defense against herbivores (Sakihama et al. 2002). In sorghum, deoxyanthocyanidin aglycones have been shown to act as phytoalexins that are synthesized and released at the site of fungal ingress (Nicholson et al. 1986; Snyder and Nicholson 1990). To our knowledge, however, anthocyanins have not been reported to serve in the molecular recognition of plants by microbes. Here, we show that the deoxyanthocyanins of Azolla can act synergistically with unknown components in frond extract or with naringin to induce hrmA-luxAB in cells of N. punctiforme UCD 328.

\section{RESULTS}

\section{Occurrence of deoxyanthocyanins and induction of hrmA-luxAB.}

Extracts from fronds of $A$. filiculoides and $A$. pinnata were screened for hrmA-luxAB-inducing activity by monitoring luminescence from luciferase (LuxAB) in cells of $N$. punctiforme strain UCD 328 following overnight incubations. All Azolla extracts induced hrmA-luxAB in a dose-dependent manner, but the intensity of induction varied according to the species and to the growth conditions of the source plants (Fig. 1). The levels of frond deoxyanthocyanins showed a significant positive correlation with hrmA-luxAB-inducing activities $(P<0.05)$. Compared to $A$. filiculoides cultivated under equivalent conditions, A. pinnata fronds had two- to threefold higher levels of hrmAluxAB-inducing activity (Fig. 1) and deoxyanthocyanins (Fig. 2A). For both Azolla species, winter-season, pond-cultivated fronds had approximately twofold higher levels of hrmAlux $A B$-inducing activity (Fig. 1) and fourfold higher levels of deoxyanthocyanins (Fig. 2A) than those of plants cultured under controlled laboratory conditions.

Deoxyanthocyanins were detectable in extracts from fronds and not from roots. Their intracellular localization was apparent in the reddish vacuoles of epidermal cells (Fig. 2B) that were not discernable in green mesophyll cells. Trichomes that arise from the epidermal cell layer of Azolla symbiotic cavities are thought to function in the exchange of metabolites between the plant and symbionts (Calvert et al. 1985; Peters and Meeks 1989). The cytoplasm of most trichome cells of $A$. pinnata had a speckled varicolored appearance (Fig. 2C) consistent with previous observations of trichomes in Azolla caroliniana cavities (Peters et al. 1978). Deoxyanthocyanins were determined to represent roughly $0.8 \%$ of winter-season $A$. pinnata frond dry mass, similar to the level of anthocyanin accumulated in red leaves of rainforest understory shade plants (Gould et al. 1995). As the end of winter approached, apical leaf growth became visible as a green ring around the frond perimeter. By midsummer, levels of deoxyanthocyanin in A. pinnata fronds had declined to approximately $14 \%$ of their peak and held at that level until rising again the following winter (Fig. 3, inset).

In accordance with a previous analysis of $A$. pinnata phenolics (Ishikura 1982), we have found no evidence for the accumulation in fronds of flavonoids other than the deoxyanthocyanins. No flavanones, including naringin, a known inducer of hrmA-luxAB, were detected on thin layer chromatographs of various Azolla extracts (data not shown). In an attempt to isolate one or more hrmA-luxAB components, aqueous phosphatebuffered ( $\mathrm{pH} 7.8$ ) extract of A. pinnata was loaded onto a column packed with Sephadex LH-20 and eluted with phosphate buffer followed by ethanol. Elutant fractions were collected and assayed with cells of strain UCD 328. Substantial hrmA-
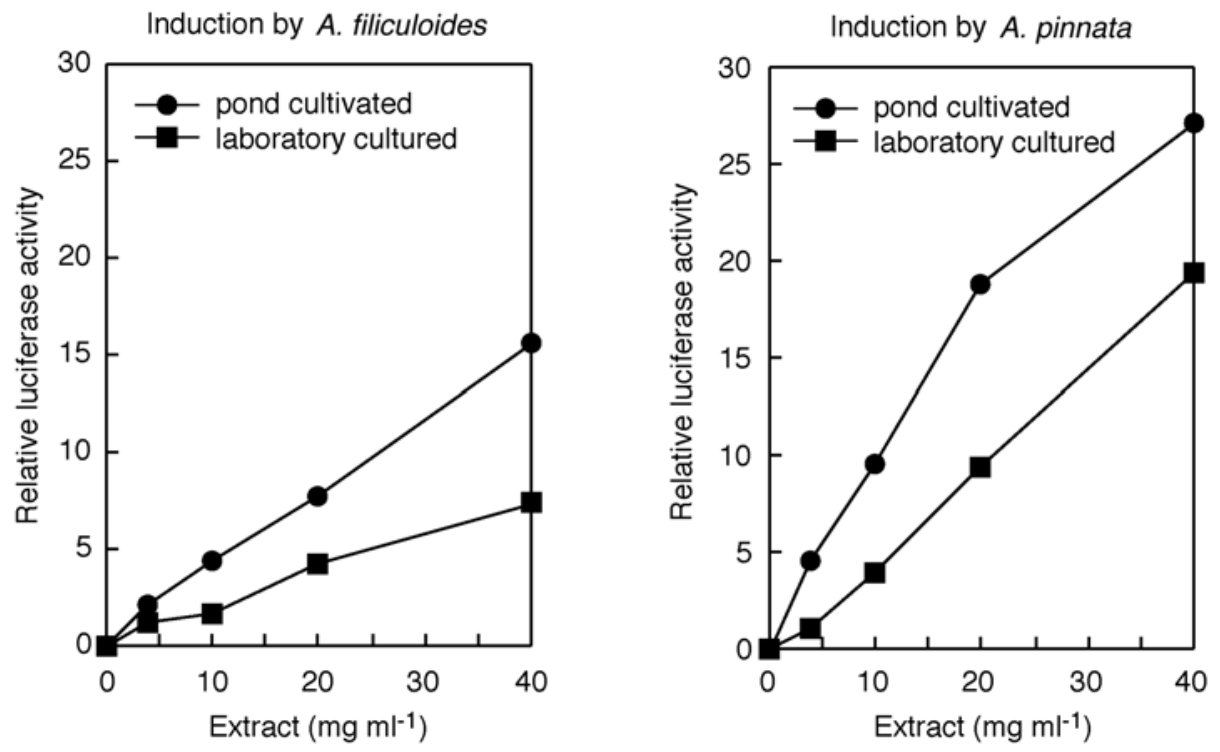

Fig. 1. Induction of hrmA-luxAB by frond extracts of Azolla filiculoides (left) and A. pinnata (right). Reported luciferase activity values are relative to light production from cells of Nostoc punctiforme strain UCD 328 incubated with growth medium only. Data points represent the means of at least two independent measurements. 
$\operatorname{lux} A B$-inducing activity ( $>2$ relative luciferase activity [RLA]) was not found in any fraction (data not shown), raising the possibility that a combination of components in frond extract is required for activity.

The observed correlation between deoxyanthocyanins in fronds and the hrmA-luxAB-inducing activity of their extracts warranted a more thorough investigation of the possible influence of these compounds on hrmA induction.

\section{Purification of deoxyanthocyanins.}

Since A. pinnata contained greater levels of pigmentation than $A$. filiculoides, we chose pond-cultivated $A$. pinnata fronds as our source of deoxyanthocyanin for assays of hrmAlux $A B$ inducing activity. Simple phenolic compounds, which absorb light in the UV but not visible range (Ishikura 1982), were the major contaminants in crude extract preparations. Relying on the differential binding of deoxyanthocyanins to $\mathrm{C}-8$ sorbent and the lack of binding to LH-20 particles, we successfully purified deoxyanthocyanins from A. pinnata. The deoxyanthocyanin preparation was considered nearly pure when its visible absorbance peak around $500 \mathrm{~nm}$ was at least 1.8 times greater than the peak around $280 \mathrm{~nm}$, and further extractions did not yield a greater ratio (Fig. 3). A positive silver nitrate reaction to the aqueous phase deoxyanthocyanin acid hydrolysate indicated substitution by one or more $O$-linked sugars (data not shown), in accordance with a previous finding of a C-5 hydrolysable glucosyl residue on the deoxyanthocyanins of $A$. pinnata (Ishikura 1982).

Figure 4 shows a high-performance liquid chromatography (HPLC) profile of the preparation purified from A. pinnata. Three peaks, one major and two minor, were detected by monitoring absorbance at $490 \mathrm{~nm}$. The HPLC profile of a preparation purified from A. filiculoides showed a very similar chromatogram (Fig. 4, inset). Separation of the A. pinnata deoxyanthocyanin preparation by thin-layer chromatography (TLC) revealed three colored bands (Table 1) that were purified from the TLC plates (discussed in detail below) and individually subjected to HPLC analysis. The major orange compound (LG1) eluted at $12.5 \mathrm{~min}$, the yellow compound (AG1) at 14 min, and the minor orange compound (LG2) at $8 \mathrm{~min}$. Storage of LG2 at room temperature resulted in a decrease in the $8 \mathrm{~min}$ peak and the development of a noticeable 10.5 peak, more apparent in the A. filiculoides chromatogram (Fig. 4), possibly due to autooxidation of the compound.

\section{Deoxyanthocyanin characterization.}

All three purified compounds showed spectra characteristic of deoxyanthocyanins. Visible absorption maxima were approximately $45 \mathrm{~nm}$ less than those corresponding to 3-oxyanthocyanins (Harborne 1958). Addition of $\mathrm{AlCl}_{3}$ to solutions of the LG1 or LG2 shifted their visible absorption peaks by approximately $+50 \mathrm{~nm}$ (Table 1), diagnostic of complex formation between $\mathrm{Al}^{3+}$ and the $\sigma$-dihydroxyl group of luteolinidin and its glycosides (Harborne 1958). Compound AG1 showed fluorescence under UV light and its spectrum was not shifted by $\mathrm{AlCl}_{3}$. Acid hydrolysis of the three compounds released aglycones having the spectral and chromatographic characteristics of apigeninidin for AG1 and luteolinidin for LG1 and LG2 (Table 1).

Based on previous chemical analyses, an unidentified glycoside of apigeninidin has been reported in A. caroliniana and A. filiculoides (Pieterse et al. 1977) and the predominant luteolinidin derivative isolated from several Azolla species, including A. pinnata, has been reported to be luteolinidin-5-glucoside (433 MW) (Harborne and Baxter 1999; Ishikura 1982). Fast atom bombardment mass spectrometry (FAB-MS) analysis on the purified deoxyanthocyanin mixture showed two predominant peaks at $\mathrm{m} / \mathrm{z} 459$ and $\mathrm{m} / \mathrm{z} 475$, which were found by HPLC-MS analysis to correspond to AG1 and LG1, respectively (Fig. 5). Determination of molecular formulas from high-resolution MS analysis gave $\mathrm{C}_{23} \mathrm{H}_{23} \mathrm{O}_{11}{ }^{+}$for LG1, consistent with an acetyl derivative of a luteolinidin glycoside, and $\mathrm{C}_{23} \mathrm{H}_{23} \mathrm{O}_{10}{ }^{+}$for $\mathrm{AG} 1$, consistent with an acetyl derivative of an apigeninidin glycoside. It is not uncommon for MS analysis of flavonoids to reveal acetyl groups previously missed by chemical analyses (Bohm 1998). Compound LG2 was not present in sufficient amounts for MS analysis.

\section{Deoxyanthocyanin-enhanced expression of hrmA-luxAB.}

Incubation of $N$. punctiforme strain UCD 328 cells with up to $150 \mu \mathrm{M}$ deoxyanthocyanins resulted in only a $70 \%$ increase
A. filiculoides

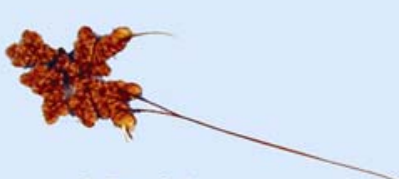

$8.5 \pm 3.1$

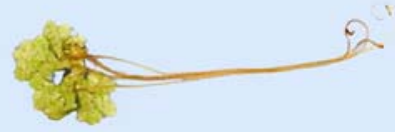

$2.1 \pm 0.8$
A. pinnata

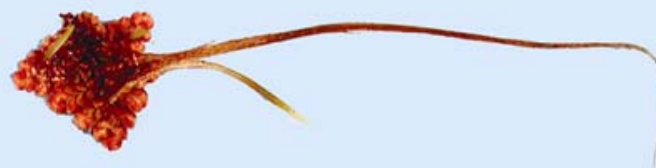

$23.9 \pm 3.5$

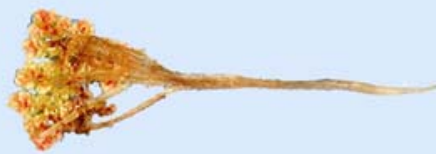

$5.6 \pm 2.1$
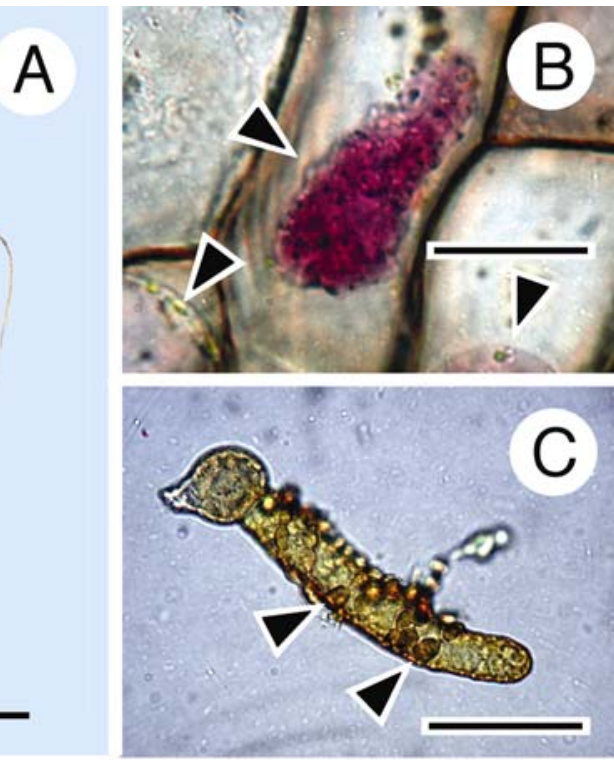

Fig. 2. A, Ventral view of Azolla spp. plants demonstrating their increased deoxyanthocyanin accumulation in outdoor ponds during winter (top) relative to plants cultured under standard laboratory conditions (bottom). Values are means \pm SE of frond tissue deoxyanthocyanin levels from at least three independent measurements in units of $A_{500} \mathrm{ml} \mathrm{g}^{-1}$. Bar $=2 \mathrm{~cm}$. B, Micrograph of epidermal cells containing reddish vacuoles (arrows). Bar $=20 \mu \mathrm{m}$. C, Micrograph of an epidermal trichome released by enzymatic digestion of fronds followed by rapid plunging through a pasteur pipette. Nostoc punctiforme cells (arrows) commonly remained attached to the trichomes. Bar $=15 \mu \mathrm{m}$. 
in hrmA-luxAB activity compared with the constitutive activity from control cells. However, addition of the deoxyanthocyanin preparation to diluted $A$. filiculoides frond extract increased the hrmA-lux $A B$-inducing activity to a significantly greater degree than theoretically expected by adding the individual inducing activities of extract and the deoxyanthocyanins (Fig. 6). A similar synergistic enhancement of hrmA-luxAB induction by deoxyanthocyanins was found in assays where A. filiculoides extract was replaced with a naringin solution (Fig. 6). Purified LG1 showed an enhancement effect at nearly the same level of activity as the deoxyanthocyanin preparation (data not shown). Partially purified preparations of other A. pinnata phenolics, containing mixtures of coumaric acid, aesculetin, chlorogenic acid, and caffeic acid derivatives (Ishikura 1982), did not show enhancement of hrmA-luxAB induction (data not shown). Growth of $N$. punctiforme cells was not inhibited by up to 250 $\mu \mathrm{M}$ deoxyanthocyanins (the highest concentration tested).

\section{DISCUSSION}

\section{Deoxyanthocyanins as potential} in planta coinducers of $h r m A$.

Our present results show that induction of hrmA-luxAB by Azolla spp. extract is due to the synergistic actions of deoxyanthocyanins and other plant-derived components. The 26-fold increase in hrmA-lux $A B$ activity induced by incubation with 40 $\mathrm{mg}$ of $A$. pinnata extract per $\mathrm{ml}$ (Fig. 1) is higher than the maxima previously reported to be induced by the common flavonoid naringin (16-fold; Cohen and Yamasaki 2000) and by extracts of A. punctatus (10-fold; Cohen and Meeks 1997) and other plants we have assayed (M. F. Cohen and H. Yamasaki, unpublished data). For the hrmA induction phenomenon to

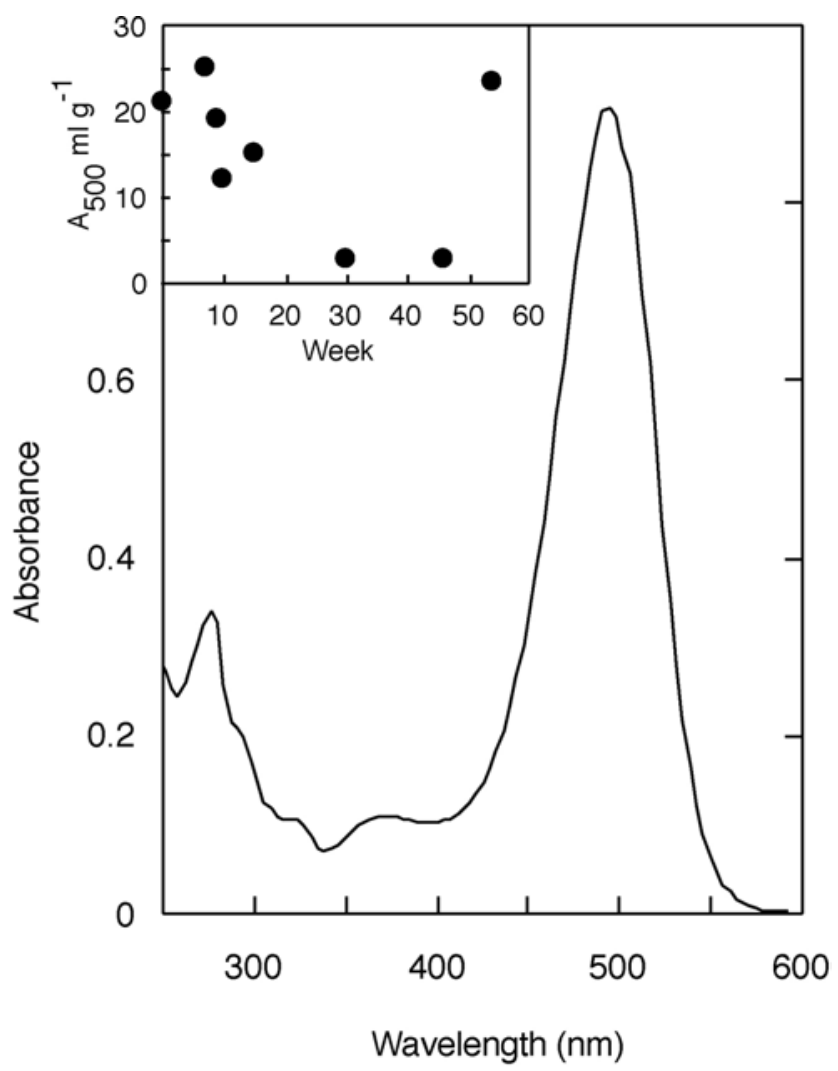

Fig. 3. Ultraviolet/visible absorbance spectrum of an Azolla pinnata purified deoxyanthocyanin preparation diluted 30 -fold in acidified methanol. The inset shows the changes in frond deoxyanthocyanin levels of pondcultivated A. pinnata over a 54-week period beginning on Jan. 28, 2000. have physiological relevance in Azolla spp., the Nostoc spp. filaments would need to come in contact with the hrmA-inducing components. The epidermal trichomes that penetrate the Azolla spp. cavities exhibit a transfer cell ultrastructure associated with sites of high solute transfer in plants (Peters and Meeks 1989) and, therefore, would represent the likely sites for symbiont exposure to putative regulatory molecules. The identity of the colored components within the trichome cells cannot be definitively established from our results, but anthocyanins and their derivatives are known to display several colors depending on $\mathrm{pH}$, solute composition, hydration, and oxidation state (Bohm 1998). While increased deoxyanthocyanin levels in Azolla spp. fronds under stress undoubtedly serves protective purposes not directly related to the symbiosis (Sakihama et al. 2002), it is also compatible with an inferred reduction in the need for hormogonia in fronds of slow-growing or dormant plants.

A role for $h r m A$ in lowering the frequency of hormogonium formation in Azolla spp. is difficult to assess directly, since the major symbiont of Azolla spp. is recalcitrant to culturing (Tang et al. 1990) and attempts at reconstituting the symbiosis with a laboratory-cultured cyanobacterium have been unsuccessful (Peters and Meeks 1989). Although it is possible that the hrmA-inducing activity in extract is not indicative of an actual function in Azolla spp. plants, the results reported here nonetheless provide insight into the more general phenomenon of $N$. punctiforme responses to plant products.

\section{Symbiosis mediation by flavonoid-containing mixtures.}

A capacity to respond to mixtures of plant compounds could help $N$. punctiforme maintain its wide host specificity. An analogous bacterial response to plant products can be found in certain rhizobia whose nod genes are induced by structurally diverse flavonoids (Hungria et al. 1992) and other compounds (Gagnon and Ibrahim 1998) released by legume plant hosts. Mixtures of some of these compounds give higher levels of nod gene induction than expected by an additive model. From our experiments, the mechanism for the synergistic induction of hrmA cannot be determined, but at least two scenarios are possible: formation of intermolecular complexes, a characteristic of anthocyanins (Bohm 1998), could produce more efficient inducers of the $\mathrm{hrm}$ operon receptors, or a variety of inducers could bind to multiple receptor types, as in rhizobia species that produce multiple NodD proteins with different inducer-

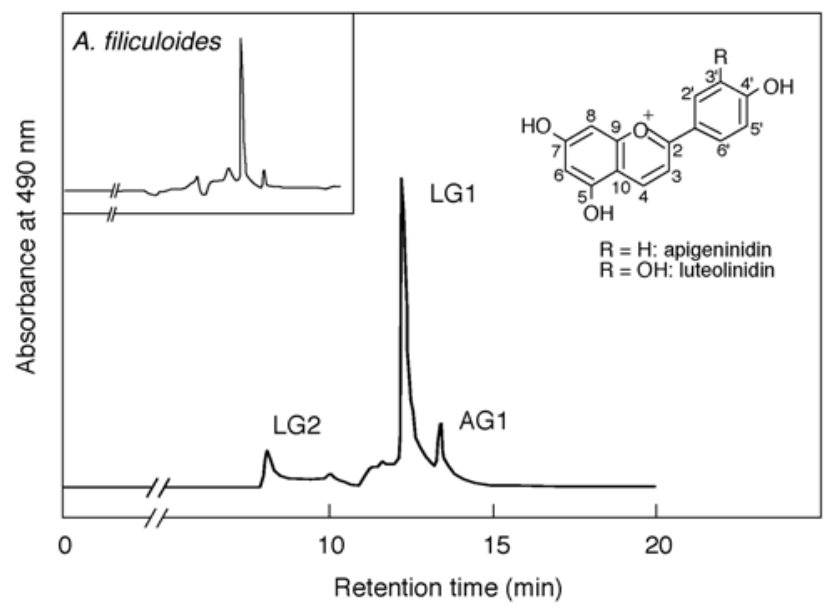

Fig. 4. High-performance liquid chromatography (HPLC) profile of an Azolla pinnata deoxyanthocyanin preparation. The inset on the top left shows a HPLC profile of an equivalent preparation from A. filiculoides. The inset on the right shows a two-dimensional representation of the deoxyanthocyanidin skeletal ring structure. 
binding specificities. Studies are currently underway to identify potential regulatory proteins that bind DNA in the vicinity of the inducible hrm operon promoters (E. L. Campbell and J. C. Meeks, personal communication).

\section{A model for hormogonium regulation in Azolla spp.}

The function, if any, of the hormogonium-like filaments in Azolla spp. symbioses has long been uncertain. That the directed movement of Nostoc spp. within Azolla spp. plants is accomplished by specialized plant epidermal trichomes may explain the immobility of these hormogonia (Peters and Meeks 1989). Evolutionary retention of the hormogonium state, however, implies that hormogonia have some utility in the Azolla spp. symbiosis. Since their surface properties differ substantially from those of vegetative filaments (Fattom and Shilo 1984; Schüßler et al. 1997), the formation of hormogonia in apical cavities has been hypothesized to enable recognition by trichomes so that only generative hormogonium cells, and not senescent or terminally differentiated cells, serve as inocula for new cavities (J. C. Meeks, personal communication). This type of discernment is displayed by Geosiphon pyriforme, a fungal host of endosymbiotic $N$. punctiforme. Hyphal tips of the fungus only incorporate $N$. punctiforme cells that are in transition from the hormogonium phase, even developing heterocysts within these nonmotile "pimordium" filaments are excluded (Mollenhauer et al. 1996).

Figure 7 depicts a model for Nostoc spp. colonization and development in Azolla spp. symbiotic cavities postulating the presence of HIF concentrated at the stem apex and hormogonium-repressing factor (HRF) more prevalent in mature leaf cavities. In free-living Nostoc spp., vegetative filaments derived from hormogonia display a temporary immunity to plant HIF (Campbell 1988) that appears to require a functional $\mathrm{hrm}$ operon (Cohen and Meeks 1997). As immunity to HIF is lost, filaments convert to hormogonia in a process that involves cell division without an accompanying increase in biomass (Campbell and Meeks 1989). Continual running of an HIF-stimulated hormogonium cycle in the Azolla spp. stem apex would account for the relatively low Nostoc spp. cell dimensions in this region (Hill 1975). Formation of hormogonia does not occur in mature cavities, conceivably due to the presence of HRF. In growing plants, mature frond tissue is more red than the actively growing apical tissue (discussed above), perhaps indicative of a role for deoxyanthocyanins as an HRF component. Cells whose growth is not interrupted by the cell divisions of hormogonium formation would be permitted to reach the larger average sizes observed by Hill (1975) in mature cavities. Import of fixed $\mathrm{N}$ from mature leaves represses the formation of heterocysts in the stem apex (Kaplan and Peters 1981). Cutoff of this fixed N delivery (Kaplan and Peters 1981), combined with exposure to putative inducers of $N$. punctiforme het-

Table 1. Spectral properties of Azolla pinnata deoxyanthocyanins and hydrolysis products and their separation by thin-layer chromatography (TLC)

\begin{tabular}{lccccc}
\hline & \multicolumn{3}{c}{$\begin{array}{c}\text { Spectral maxima } \\
\lambda_{\text {max }}(\mathbf{n m})\end{array}$} & & \\
\cline { 2 - 3 } Compound & $\mathbf{U V}$ & Visible & & \multicolumn{3}{c}{ shift } & \\
\hline Orange compound, LG1 & 498 & 278 & 54 & 0.79 \\
Hydrolysis product & 501 & 282 & 49 & 0.67 \\
Orange compound, LG2 & 495 & 277 & 55 & 0.85 \\
Hydrolysis product & 496 & 278 & 50 & 0.67 \\
Yellow compound, AG1 & 481 & 277 & 0 & 0.88 \\
Hydrolysis product & 479 & 278 & 0 & 0.83 \\
Apigeninidin & 479 & 278 & 0 & 0.83 \\
Luteolinidin $^{\mathrm{b}}$ & 496 & 279 & 52 & 0.64 \\
\hline
\end{tabular}

${ }^{a}$ TLC solvent, acetic acid-HCl- $\mathrm{H}_{2} \mathrm{O}$ (30:3:10) for all except luteolinidin.

${ }^{\mathrm{b}}$ Values from Harborne (1958, 1966); TLC solvent, ethyl acetate-formic acid-2 $\mathrm{M} \mathrm{HCl}(85: 9: 6)$. erocyst differentiation, may be responsible for the high frequency of heterocysts (approximately 30\%) in mature cavities (Hill 1975).

\section{Implications for other plant- $N$. punctiforme symbioses.}

We have shown that the hrmA gene of $N$. punctiforme can be induced by a combination of components, including deoxyanthocyanins, in Azolla spp. frond extract (Figs. 1 and 6). The low host-specificity characteristic of $N$. punctiforme implies that our results will have relevance to other plant-Nostoc spp. symbioses, all of which appear to involve some level of host control over hormogonium formation. A role for the hrm operon in symbiotic hormogonium regulation may be common among Nostoc spp., since seven out of seven Nostoc strains tested showed DNA hybridization to hrmU and hrmA probes (Cohen 1996).

Though anthocyanins are widely distributed among the plant kingdom, they have not been previously reported to serve as molecules of plant recognition by symbionts. This may be due to their occurrence mainly in aerial plant structures and not in roots, where most symbionts reside. The ecological niche of Nostoc spp. encompasses fresh water, soil, and both the internal and external surfaces of plant hosts (Dodds et al. 1995). Thus, it is not unexpected that a symbiotic-related gene of Nostoc spp., such as hrmA, should be responsive to plant anthocyanin. In the more intimate plant-Nostoc spp. symbioses, the composition of certain tissues may be tailored to encourage the natural tendency of plant-associated Nostoc spp. to repress hormogonium formation and form adherent colonies.

Presumptive accumulations of anthocyanin in hosts other than Azolla spp. are found in regions proximate to Nostoc spp. filaments: in Gunnera spp., bright red symbiotic stem glands are covered in an HIF-containing mucilage (Rasmussen et al. 1994) and in cycads, symbiotic coralloid roots are delimited by pinkish phenolic compounds at their basal boundary (Lobakova
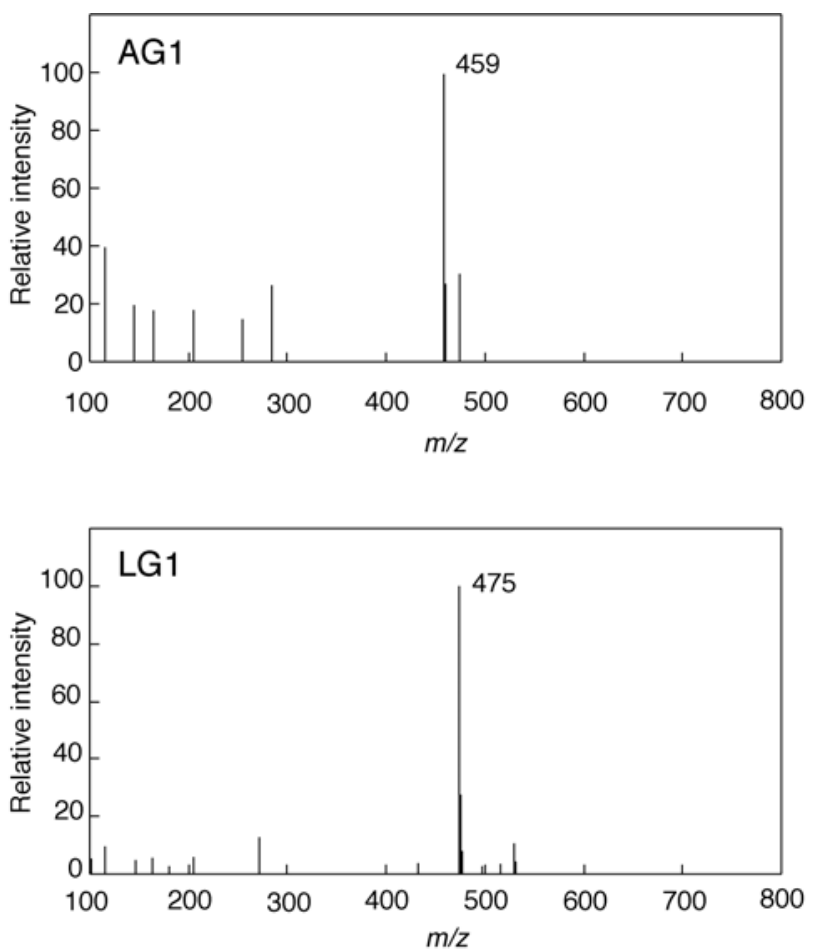

Fig. 5. Atmospheric pressure chemical ionization positive ion mass spectra of compounds AG1 (top) and LG1 (bottom). Intensity readings are normalized to the major peak in each spectrum. To lessen signal noise, values lower than 3 and 15 normative units have been excluded from the LG1 and AG1 spectra, respectively. 
et al. 2001). Since the Gunnera spp. and cycad symbioses with Nostoc spp. can be reconstituted, they may represent useful experimental systems for examining possible roles of anthocyanins in the localized counteraction of HIF.

\section{MATERIALS AND METHODS}

\section{Growth conditions.}

A. filiculoides IRRI FI1090 was obtained from N. Shiomi of the University of Osaka. A. pinnata was isolated from a local taro field in Okinawa, a subtropical island of southern Japan. Plants were cultured in the laboratory at 23 to $25^{\circ} \mathrm{C}$ in bubbled medium, with an $18 \mathrm{~h}$ light and $6 \mathrm{~h}$ dark cycle using a combination of fluorescent and incandescent light $\left(100 \mu \mathrm{mol} \mathrm{m}^{-2} \mathrm{~s}^{-1}\right)$. The culture medium contained $0.5 \mathrm{mM} \mathrm{MgSO}_{4}, 2 \mathrm{mM} \mathrm{CaCl}_{2}, 1 \mathrm{mM}$ $\mathrm{K}_{2} \mathrm{SO}_{4}, 0.6 \mathrm{mM} \mathrm{Na}_{2} \mathrm{HPO}_{4}, 0.4 \mathrm{mM} \mathrm{KH} \mathrm{PO}_{4}$, and two stock solutions of Allen and Arnon (1955): $1 \mathrm{ml}$ of Fe-EDTA and $1.5 \mathrm{ml}$ of microelements per liter. A. filiculoides was cultured in fullstrength medium, while $A$. pinnata was cultured in medium with the phosphate salts and Fe-EDTA solution diluted twofold and all other components diluted 20-fold. Plants were also maintained outdoors under partial shade in unsupplemented $75 \times 45 \times 60 \mathrm{~cm}$ cement-lined ponds ( $\left.\mathrm{pH} 7.3 ; 30 \mu \mathrm{M} \mathrm{PO}_{4}{ }^{3-}\right)$. Chemical analyses were conducted on deoxyanthocyanins from winter season $\left(20^{\circ} \mathrm{C}\right.$ maximum and $14^{\circ} \mathrm{C}$ minimum mean daily air temperatures) pond-cultivated plants. Nostoc spp. cells were cultured under conditions previously described (Cohen et al. 1994).

\section{Deoxyanthocyanin quantification.}

$\mathrm{Up}$ to $15 \mathrm{~g}$ derooted frond tissue was ground in liquid nitrogen and sequentially extracted, twice with $10 \mathrm{ml}$ of $5 \% \mathrm{H}_{3} \mathrm{PO}_{4}$ per $\mathrm{g}$ of fresh weight and twice with $4 \mathrm{ml}$ of acidified $(1 \% \mathrm{HCl})$ methanol per $\mathrm{g}$ of equivalent fresh weight. The methanolic portion was concentrated under vacuum to a minimal volume $(<10$ $\mathrm{ml})$ and diluted 10 -fold with acidified $(0.1 \% \mathrm{HCl})$ water. The dilutant was combined with the aqueous $\mathrm{H}_{3} \mathrm{PO}_{4}$ extract and filtered through Whatman no. 2 paper and a $3.0-\mu \mathrm{m}$ pore size cellulose acetate filter. The filtrate was then passed through C-8 and C-18
Sep-Pak cartridges (Waters, Milford, MA, U.S.A.) connected in series, which had been pretreated with successive applications of $10 \mathrm{ml}$ of acetone, $10 \mathrm{ml}$ of methanol, and $5 \mathrm{ml}$ of acidified water. All deoxyanthocyanins were retained on the cartridges. Following a rinse with $5 \mathrm{ml}$ of acidified water, the deoxyanthocyanins were eluted with acidified $(0.01 \% \mathrm{HCl})$ methanol. For comparisons, the total tissue deoxyanthocyanin concentrations were calculated as $\mathrm{A}_{500} \mathrm{ml}$ of elutant per $\mathrm{g}$ of fresh weight.

\section{Spectral and chromatographic analyses.}

Absorbance spectra were measured on a Shimadzu UV1650PC UV/visible spectrophotometer. To determine spectral shifts, $5 \% \mathrm{AlCl}_{3}$ in methanol was added at a 1:10 ratio, and the spectrum measured after 3 min.

Extracts and solutions of purified compounds were analyzed by TLC on SF-microcellulose plates (Funakoshi, Tokyo, Japan). The solvent systems were acetic acid-HCl- $\mathrm{H}_{2} \mathrm{O}$ (30:3:10) or $n$-butanol-acetic acid-water (4:1:5). To test for flavanones, plates were sprayed with a $2 \%$ solution of $\mathrm{NaBH}_{4}$ in methanol and fumed for 5 min with $\mathrm{HCl}$ (Grayer 1989). The naringenin and naringin positive controls gave red and purple color, respectively.

HPLC analysis was carried out with a reverse-phase C-18 column (5C18 ARII, $4.6 \times 50 \mathrm{~mm}$; Nacalai Tesque, Kyoto, Japan). Solvent A was $7.5 \%$ acetonitrile in water containing $5 \%$ acetic acid, and solvent B was $100 \%$ methanol. The elution program was a linear gradient from 0 to $100 \%$ solvent B over $20 \mathrm{~min}$ at a flow rate of $0.5 \mathrm{ml} \mathrm{min}{ }^{-1}$. Peaks were monitored at $490 \mathrm{~nm}$ and recorded with a Chromatopac integrator (C-R3A; Shimadzu, Kyoto, Japan).

HPLC peaks were analyzed by atmospheric pressure chemical ionization (APCI)-MS with a Waters 2690 Alliance HPLC linked to a JEOL (Tokyo) JMS-700 mass spectrophotometer. All spectra were obtained in the positive-ion mode over an $\mathrm{m} / \mathrm{z}$ range of 10 to 1,000 at one scan every $3 \mathrm{~s}$ and were collected in the form of continuum data. The HPLC separations were carried out using a Cadenza CD-C18 column $(50 \times 4.6 \mathrm{~mm}$ inner diameter, Intakt Co., Kyoto, Japan). The HPLC solvents and
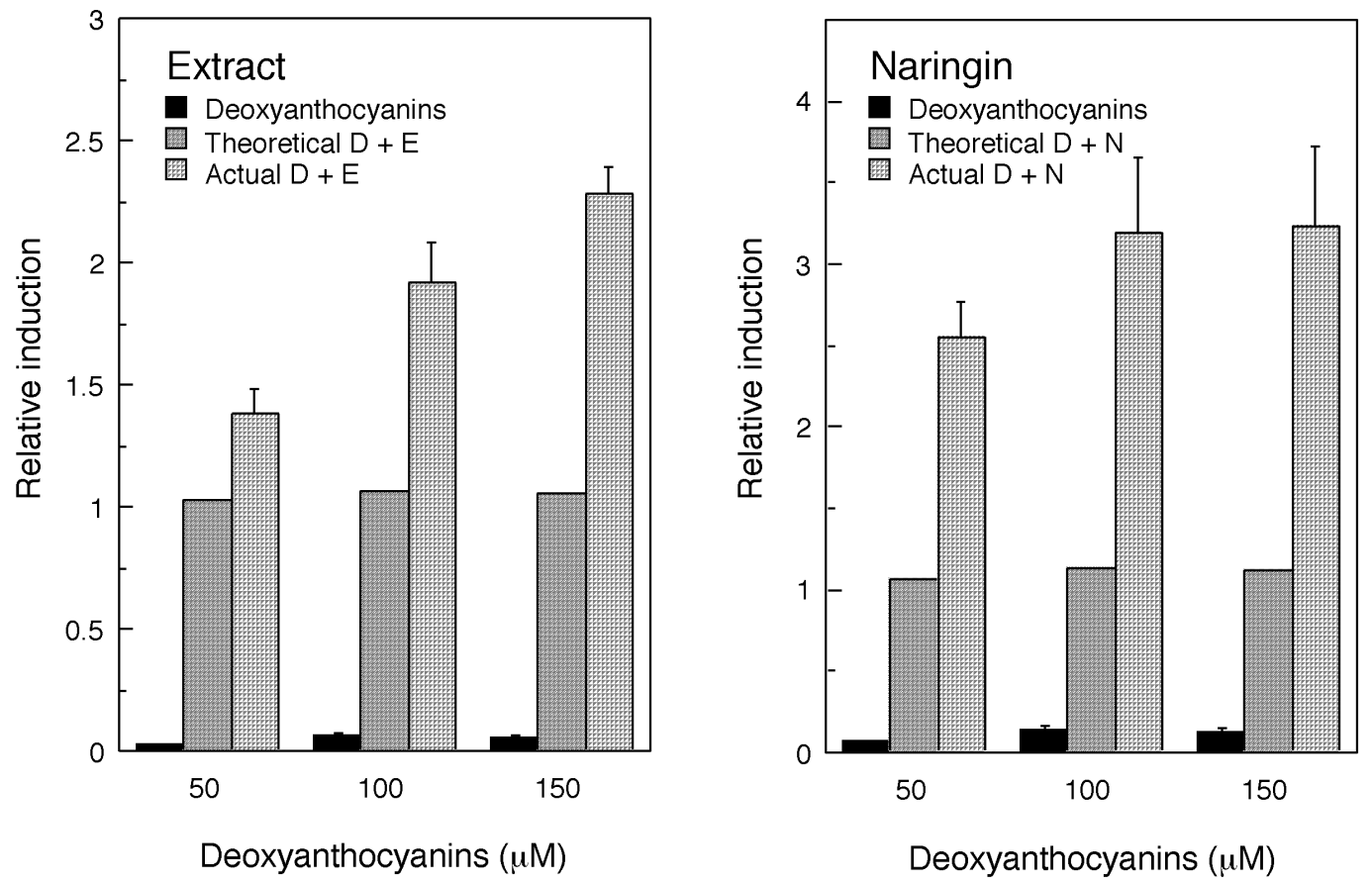

Fig. 6. Synergistic enhancement of $h r m A-l u x A B$ induction by deoxyanthocyanins. Values are reported relative to the induction by solutions of $23 \mathrm{mg} \mathrm{ml}^{-1}$ laboratory-cultured Azolla filiculoides frond extract (left) and $100 \mu \mathrm{M}$ naringin (right) without added deoxyanthocyanins. All incubations were carried out in the presence of $1 \%$ ethanol. Means $\pm \mathrm{SE}$ are from at least three independent experiments. $\mathrm{D}=$ deoxyanthocyanins, $\mathrm{E}=$ extract, and $\mathrm{N}=$ naringin . 
elution program were the same as described above, except for using a flow rate of $1.0 \mathrm{ml} \mathrm{min}{ }^{-1}$. The deoxyanthocyanin preparation was also analyzed as a mixture by FAB-MS with glycerol as the matrix.

\section{Deoxyanthocyanin purification.}

HPLC analysis showed that extraction of fronds with either aqueous $5 \% \mathrm{H}_{3} \mathrm{PO}_{4}$ or acidified $(1 \% \mathrm{HCl})$ methanol yielded the same deoxyanthocyanins in similar proportions (data not shown). For the purpose of deoxyanthocyanin purification, fronds were extracted only with aqueous $5 \% \mathrm{H}_{3} \mathrm{PO}_{4}$, since methanol solubilizes excessive amounts of phenolic contaminants. The extract was filtered, and the filtrate combined with Sephadex LH-20 (1 g per $10 \mathrm{ml}$ of filtrate) (Pharmacia, Uppsala, Sweden) and stirred for $1 \mathrm{~h}$ at room temperature. The Sephadex LH-20 with its absorbed contaminants was removed from the solution by filtration and the filtrate was passed through a C-8 Sep-Pak cartridge. The cartridge with the absorbed sample was washed with acidified water and then rinsed with $5 \mathrm{ml}$ ethyl acetate to selectively elute polyphenolics other than the deoxyanthocyanins (Skrede et al. 2000). The deoxyanthocyanins were eluted from the cartridge with acidified $(0.01 \%)$ methanol.

For purification of the individual deoxyanthocyanins, the elutant was evaporated in vacuum to a minimal volume and was subjected to preparative TLC (Harborne 1989). Colored bands were scraped into microfuge tubes and were eluted with acidified methanol, and their purity determined by measuring absorbance spectra and by HPLC analysis.

\section{Deoxyanthocyanidin preparation.}

Acid hydrolysis of the deoxyanthocyanins was carried out in $2 \mathrm{M} \mathrm{HCl}$ at $100^{\circ} \mathrm{C}$ for $90 \mathrm{~min}$ (Harborne 1989). The deoxyanthocyanidin (aglycone) products were collected by phase separation into isoamyl alcohol, the solvent evaporated under a stream of $\mathrm{N}_{2}$, and the pigments resuspended in acidified $(0.01 \% \mathrm{HCl})$ methanol. To confirm the release of sugar residues, the aqueous phase hydrolysate was spotted onto a TLC plate. The solvent system was ethyl acetate-acetic acid-water (14:3:3). After drying, the plate was sprayed with $0.1 \mathrm{M}$ $\mathrm{AgNO}_{3}$ followed by $0.5 \mathrm{M} \mathrm{NaOH}$ in $80 \% \mathrm{EtOH}$. Development of a brown color indicated the presence of sugar (Lewis and Harley 1965).

Synthesis of the apigeninidin standard was performed as described by Nicholson et al. (1986), except the flavan-4-ol intermediate and the final products were both concentrated on C-8 and C-18 Sep-Pak cartridges connected in series, instead of extracting the compounds into organic solvents.

\section{Luciferase assays.}

RLA, normalized to the concentration of strain UCD 328 cells, was assayed as previously described (Cohen and Yamasaki 2000). Since deoxyanthocyanins absorb strongly in range of LuxAB light emission (490 nm), cells were collected by centrifugation and resuspended in fresh medium immediately before measuring luminescence. Aqueous Azolla spp. extracts were prepared for luciferase assays by homogenizing derooted fronds in $10 \mathrm{ml}$ of $N$. punctiforme growth medium per $\mathrm{g}$ of fresh weight and then centrifuging at 3,000 $\mathrm{g}$ for $10 \mathrm{~min}$. The supernatant was combined with cells of strain UCD 328 and was incubated under light for 14 to $16 \mathrm{~h}$. The significance of the coefficient of correlation $(r)$ between frond deoxyanthocyanin levels and $h r m A$-inducing activities was determined by employing a one-tailed test of the following computed test statistic.

$$
t=r \frac{\sqrt{(n-2)}}{\sqrt{\left(1-r^{2}\right)}}
$$

For assays of purified deoxyanthocyanin preparations, the acidified $(0.01 \% \mathrm{HCl})$ methanol solvent was removed by first diluting 50-fold with 5\% phosphoric acid and then absorbing the deoxyanthocyanins onto a C-8 Sep-Pak cartridge. The pigment was eluted with acidified $(0.01 \% \mathrm{HCl})$ ethanol, and the absorbance spectrum determined. Aliquots were then distributed to tubes for incubation with cells of strain UCD 328. Before adding $N$. punctiforme growth medium and cells to the tubes, the solvent was vacuum-evaporated and the deoxyantho-
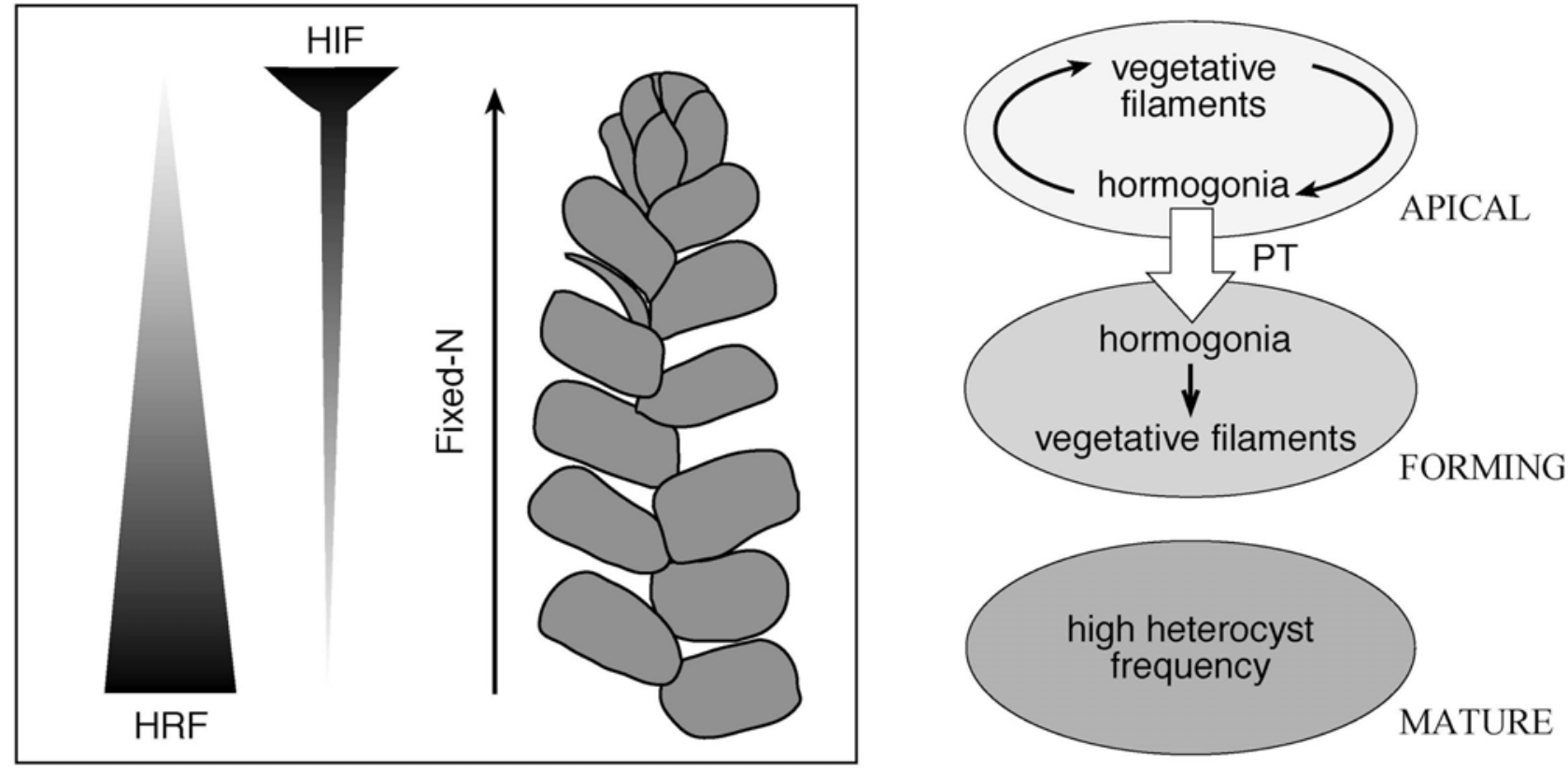

Fig. 7. A model for Nostoc spp. developmental regulation in relation to leaf maturation. Theoretical gradients of one or more putative hormogonium inducing factors (HIF) and one or more hormogonium repressing factors (HRF) are indicated next to a sketch of an Azolla pinnata main stem axis having all lateral branches removed (left). Nostoc spp. developmental forms in the pocket formed by budding leaves and apical meristem and in the cavities of forming leaves and of mature leaves (right). PT = a specialized epidermal partioning trichome that extends from a forming leaf cavity to obtain a hormogonia inoculum from the apical Nostoc spp. colony. 
cyanins resuspended in $100 \%$ ethanol, such that the final concentration for all assays would be $1 \%$ ethanol. For assays of deoxyanthocyanin enhancement of hrmA-luxAB-inducing activity, either diluted Azolla spp. extract replaced the Nostoc spp. growth medium, or an ethanolic $10 \mathrm{mM}$ naringin solution replaced ethanol.

\section{ACKNOWLEDGMENTS}

We thank E. L. Campbell and J. C. Meeks for sharing unpublished results. Support to M. F. C. and Y. S. were provided by fellowships from the Japanese Society for the Promotion of Science (JSPS). Funding was also provided to H. Y. by Grant-in-Aid for Scientific Research on Priority Areas (C) Genome Science from the Ministry of Education, Science, Sports and Culture of Japan.

\section{LITERATURE CITED}

Allen, M. B., and Arnon, D. I. 1955. Studies on nitrogen-fixing bluegreen algae. I. Growth and nitrogen fixation by Anabaena cylindrica Lemm. Plant Physiol. 30:366-372.

Bohm, B. A. 1998. Introduction to flavonoids.: Harwood Academic. New York.

Calvert, H. E., Pence, M. K., and Peters, G. A. 1985. Ultrastructural ontogeny of leaf cavity trichomes in Azolla implies a functional role in metabolite exchange. Protoplasma 129:10-27.

Camm, E. L., McCallum, J., Leaf, E., and Koupai-Abyazani, M. R. 1993. Cold-induced purpling of Pinus contorta seedlings depends on previous daylength treatment. Plant Cell Environ. 16:761-764.

Campbell, E. L. 1988. Characteristics of hormogonia formation and heterocyst differentiation in symbiotic Nostoc spp. in response to Anthoceros punctatus or its extracellular products. M.S. Thesis, University of California, Davis.

Campbell, E. L., and Meeks, J. C. 1989. Characteristics of hormogonia formation by symbiotic Nostoc spp. in response to the presence of Anthoceros punctatus or its extracellular products. Appl. Environ. Microbiol. 55:125-131.

Cohen, M. F. 1996. Transposon mutagenesis and characterization of a hormogonium regulating locus, hrmUA, of the cyanobacterium Nostoc punctiforme strain ATCC 29133. Ph.D. Dissertation, University of California, Davis.

Cohen, M. F., and Meeks, J. C. 1997. A hormogonium regulating locus, hrmUA, of the cyanobacterium Nostoc punctiforme strain ATCC 29133 and its response to an extract of a symbiotic plant partner Anthoceros punctatus. Mol. Plant-Microbe Interact. 10:280-289.

Cohen, M. F., and Yamasaki, H. 2000. Flavonoid-induced expression of a symbiosis-related gene in the cyanobacterium Nostoc punctiforme. J. Bacteriol. 182:4644-4646.

Cohen, M. F., Wallis, J. G., Campbell, E. L., and Meeks, J. C. 1994. Transposon mutagenesis of Nostoc sp. strain ATCC 29133, a filamentous cyanobacterium with multiple differentiation alternatives. Microbiology 140:3233-3240.

Cohen, M. F., Meeks, J. C., Cai, Y. A., and Wolk, C. P. 1998. Transposon mutagenesis of heterocyst-forming filamentous cyanobacteria. Methods Enzymol. 297:3-17.

Cohen, M. F., Sakihama, Y., and Yamasaki, H. 2001. Roles of plant flavonoids in interactions with microbes: From protection against pathogens to the mediation of mutualism. Pages 157-173 in: Recent Research Developments in Plant Physiology 2. S. G. Pandalai, ed. Research Signpost, Trivandrum, India.

Dodds, W. K., Gudder, D. A., and Mollenhauer, D. 1995. The ecology of Nostoc. J. Phycol. 31:2-18.

Enderlin, C. S., and Meeks, J. C. 1983. Pure culture and reconstitution of the Anthoceros-Nostoc symbiotic association. Planta 158:157-165.

Fattom, A., and Shilo, M. 1984. Hydrophobicity as an adhesion mechanism of benthic cyanobacteria. Applied Environ. Microbiol. 47:135-143

Gagnon, H., and Ibrahim, R. K. 1998. Aldonic acids: A novel family of nod gene inducers of Mesorhizobium loti, Rhizobium lupini, and Sinorhizobium meliloti. Mol. Plant-Microbe Interact. 11:988-998.

Gantar, M., Kerby, N. W., and Rowell, P. 1993. Colonization of wheat (Triticum vulgare $\mathrm{L}$.) by $\mathrm{N}_{2}$-fixing cyanobacteria: III. The role of a hormogonia-promoting factor. New Phytol. 124:505-513.

Gould, K. S., Kuhn, D. N., Lee, D. W., and Oberbauer, S. F. 1995. Why leaves are sometimes red. Nature 378:241-242.

Grayer, R. J. 1989. Flavanoids. Pages 283-323 in: Plant Phenolics. J. B. Harborne, ed. Academic Press, London.

Harborne, J. B. 1958. Spectral methods of characterizing anthocyanins.
Phytochemistry 70:22-28

Harborne, J. B. 1966. Comparative biochemistry of flavonoids. II. 3desoxyanthocyanins and their systematic distribution in ferns and gesnerads. Phytochemistry 5:589-600.

Harborne, J. B. 1989. General procedures and measurement of total phenolics. Pages 1-28 in: Plant Phenolics. J. B. Harborne, ed. Academic Press, London.

Harborne, J. B., and H. Baxter, eds. 1999. The Handbook of Natural Flavonoids. Vol. 2. John Wiley \& Sons. Chichester, U.K.

Hill, D. J. 1975. The pattern of development of Anabaena in the AzollaAnabaena symbiosis. Planta 122:179-184

Hungria, M., Johnston, A. W. B., and Phillips, D. A. 1992. Effects of flavonoids released naturally from bean (Phaseolus vulgaris) on nodDregulated gene transcription in Rhizobium leguminosarum bv. phaseoli. Mol. Plant-Microbe Interact. 5:199-203.

Ishikura, N. 1982. 3-desoxyanthocyanin and other phenolics in the water fern Azolla. Bot. Mag. Tokyo 95:303-308.

Johansson, C., and Bergman, B. 1994. Reconstitution of the symbiosis of Gunnera manicata Linden: Cyanobacterial specificity. New Phytol. 126:643-652

Kaplan, D., and Peters, G. A. 1981. The Azolla-Anabaena azollae relationship. X. ${ }^{15} \mathrm{~N}_{2}$ fixation and transport in main stem axes. New Phytol. 89:337-346.

Lewis, D. H. and Harley, J. L. 1965. Carbohydrate physiology of mycorrhizal roots of beech. I. Identity of endogenous sugars and utilization of exogenous sugars. New Phytol. 64:224-237.

Lobakova, E. S., Dol'nikova, G. A., and Korzhenevskaya, T. G. 2001. Cyanobacterial-bacterial complexes in plant syncyanoses. Mikrobiologiya 70:111-116

Meeks, J. C. 1998. Symbiosis between nitrogen-fixing cyanobacteria and plants. Bioscience 48:266-276.

Meeks, J. C., Campbell, E., Hagen, K., Hanson, T., Hitzeman, N., and Wong, F. 1999. Developmental alternatives of symbiotic Nostoc punctiforme in response to its plant partner Anthoceros punctatus. Pages 665678 in: The Phototrophic Prokaryotes. G. A. Peschek, W. Loffelhardt, and G. Schmetterer, eds. Plenum Publishing Corp., New York.

Mollenhauer, D., Mollenhauer, R., and Kluge, M. 1996. Studies on initiation and development of the partner association in Geosiphon pyriforme (Kutz) v Wettstein, a unique endocytobiotic system of a fungus (Glomales) and the cyanobacterium Nostoc punctiforme (Kutz) Hariot. Protoplasma 193:3-9.

Nicholson, R. L., Kollipara, S. S., Vincent, J. R., Lyons, P. C., and Cadena-Gomez, G. 1986. Phytoalexin synthesis by the sorghum mesocotyl in response to infection by pathogenic and nonpathogenic fungi. Proc. Natl. Acad. Sci. U.S.A. 84:5520-5524

Peters, G. A., and Meeks, J. C. 1989. The Azolla-Anabaena symbiosis: Basic biology. Ann. Rev. Plant Physiol. Plant Mol. Biol. 40:193-210.

Peters, G. A., Toia, R. E., Jr., Raveed, D., and Levine, N. J. 1978. The Azolla-Anabaena azollae relationship. VI. Morphological aspects of the association. New Phytol. 80:583-593.

Pieterse, A. H., de Lange, and van Vliet, J. P. 1977. A comparative study of Azolla in the Netherlands. Acta Bot. Neerl. 26:433-449.

Rai, A. N., Soderback E., and Bergmann, B. 2000. Cyanobacterium-plant symbioses. New Phytol. 147:449-481.

Rasmussen, U., Johansson, C., and Bergman, B. 1994. Early communication in the Gunnera-Nostoc symbiosis: Plant-induced cell differentiation and protein synthesis in the cyanobacterium. Mol. Plant-Microbe Interact. 7:696-702.

Sakihama, Y., Cohen, M. F., Grace, S. C., and Yamasaki, H. 2002. Plant phenolic antioxidant and prooxidant activities: Phenolics-induced oxidative damage mediated by metals in plants. Toxicology 47:67-90

Schüßler, A., Meyer, T., Gehrig, H., and Kluge, M. 1997. Variations of lectin binding sites in extracellular glycoconjugates during the life cycle of Nostoc punctiforme, a potentially endosymbiotic cyanobacterium. Eur. J. Phycol. 32:233-239

Skrede, G., Wrolstad, R. E., and Durst, R. W. 2000. Changes in anthocyanins and polyphenolics during juice processing of highbush blueberries (Vaccinium corymbosum L.). J. Food Sci. 65:357-364.

Snyder, B. A., and Nicholson, R. L. 1990. Synthesis of phytoalexins in sorghum as a site-specific response to fungal ingress. Science 248:1637-1639.

Tang, L. F., Watanabe, I., and Liu, C. C. 1990. Limited multiplication of symbiotic cyanobacteria of Azolla spp. on artificial media. Appl. Environ. Microbiol. 56:3623-3626.

Wagner, G. M. 1997. Azolla: A review of its biology and utilization. Bot. Rev. 63:1-26.

Yamasaki, H. 1997. A function of color. Trends Plant Sci 2:7

Yamasaki, H., Uefuji, H., and Sakihama, Y. 1996. Bleaching of the red anthocyanin induced by superoxide radical. Arch. Biochem. Biophys. 332:183-186. 